\title{
Phd Symposium Preface
}

\author{
Quan Z. Sheng ${ }^{1}$, Cesare Pautasso ${ }^{2}$, and Sonia Ben Mokhtar ${ }^{3}$ \\ ${ }^{1}$ The University of Adelaide, Australia \\ 2 The University of Lugano, Switzerland \\ ${ }^{3}$ LIRIS, CNRS, France \\ qsheng@cs.adelaide.edu.au, Cesare.pautasso@usi.ch, \\ sonia.ben-mokhtaraliris.cnrs.fr
}

Service oriented computing (SOC) has rapidly transformed from a vision, in the beginning of the century, to reality with technologies such as Web services, Cloud services, and the Internet of Things. While this has provided the industry and practitioners with the opportunities for a new generation of products and services, it has also raised many fundamental research challenges and open issues. The International Conference on Service Oriented Computing (ICSOC) is a premier annual event for researchers, practitioners and industry leaders to discuss and share the success and achievements in this vibrant and rapidly expanding area.

The ICSOC PhD Symposium, as part of the ICSOC conference, is an international forum for $\mathrm{PhD}$ students working in the broad areas of service computing, Web services and service engineering to present and discuss emerging research problems and ideas on how to tackle these issues. The goals of the ICSOC PhD Symposium event are:

- To bring together PhD students and established researchers in the field of service oriented computing and related areas,

- To enable PhD students to interact with other $\mathrm{PhD}$ students and to stimulate an exchange of ideas, suggestions, and experiences among participants,

- To give PhD students the opportunity to present and discuss their research in a constructive and critical atmosphere,

- To provide students with fruitful feedback and advice on their research and thesis proposals.

To achieve these goals, the symposium operates in a workshop format, giving $\mathrm{PhD}$ students an opportunity to showcase their research and providing them with ample feedback from senior international researchers and peer $\mathrm{PhD}$ students. This year, for the first time, we successfully experimented with a pair-presentation format, where $\mathrm{PhD}$ students introduced one another and had to prepare questions for each other in advance.

The symposium in Paphos, Cyprus is the $7^{\text {th }} \mathrm{PhD}$ Symposium of a series held without interruption in conjunction with the ICSOC conferences since 2005. This year we received 18 submissions from 10 countries. Each paper has been reviewed by at least three members of the Program Committee. The submissions were evaluated 
based on originality (novelty), problem significance, technical/scientific quality, and presentation/style. Each reviewer also provided detailed suggestions intended for improving the research work of the students. The Program Committee eventually selected 12 papers for the presentation and discussion in the symposium. The selected papers cover a wide range of topics in SOC from service engineering, quality of service, privacy and trust to service modelling, service composition, as well as emerging areas such as Web of Things and Cloud computing.

We thank all authors for their submissions and their active participation and the Program Committee members for their excellent work. We would also like to thank IBM Research, USA that has generously offered financial support to the symposium. We specifically thank Francisco Curbera from IBM Research for his continuous support of this event.

December 2011

Quan Z. Sheng

Cesare Pautasso

Sonia Ben Mokhtar

\section{Program Committee}

Boualem Benatallah
Djamal Benslimane
Walter Binder
Athman Bouguettaya
Mauro Caporuscio
Florian Daniel
Marlon Dumas
Nikolaos Georgantas
Mohand-Said Hacid
Xitong Li
Kwei-Jay Lin
Pascal Poizat
Aviv Segev
Jian Yu

Boualem Benatallah

University of New South Wales, Australia

University of Lyon, France

University of Lugano, Switzerland

CSIRO ICT Centre, Australia

Politecnico di Milano, Italy

University of Trento, Italy

University of Tartu, Estonia

INRIA Paris, France

LIRIS, France

MIT, USA

University of California, Irvine, USA

University of Evry Val d'Essonne, France

KAIST, Korea

Swinburne University of Technology, Australia 